\title{
Clinical predictors for Legionella in patients presenting with community-acquired pneumonia to the emergency department Rico Fiumefreddo $^{\dagger 1}$, Roya Zaborsky ${ }^{\dagger 1}$, Jeannine Haeuptle ${ }^{\dagger 1}$, Mirjam Christ- Crain ${ }^{1}$, Andrej Trampuz ${ }^{2}$, Ingrid Steffen ${ }^{3}$, Reno Frei ${ }^{2}$, Beat Müller ${ }^{* 4}$ and Philipp Schuetz ${ }^{1}$
}

Address: ${ }^{1}$ Department of Internal Medicine, University Hospital Basel, Basel, Switzerland, ${ }^{2}$ Departement of Infectious Diseases and Hospital Epidemiology, University Hospital Basel, Basel, Switzerland, ${ }^{3}$ Institute of Medical Microbiology, University of Basel, Basel, Switzerland and ${ }^{4}$ Department of Internal Medicine, Kantonsspital Aarau, Tellstrasse, CH-5001 Aarau, Switzerland

Email: Rico Fiumefreddo - R.Fiumefreddo@stud.unibas.ch; Roya Zaborsky - roya_z@yahoo.com; Jeannine Haeuptle - Jeannine.Haeuptle@stud.unibas.ch; Mirjam Christ-Crain -Christmj@uhbs.ch; Andrej Trampuz - TrampuzA@uhbs.ch; Ingrid Steffen - Ingrid.steffen@unibas.ch; Reno Frei - FreiR@uhbs.ch; Beat Müller* - Happy.Mueller@unibas.ch; Philipp Schuetz - Schuetzp@uhbs.ch

* Corresponding author †Equal contributors

Published: 19 January 2009

BMC Pulmonary Medicine 2009, 9:4 doi:10.1 I86/I47/-2466-9-4
Received: 17 October 2008

Accepted: 19 January 2009

This article is available from: http://www.biomedcentral.com/I47/-2466/9/4

(C) 2009 Fiumefreddo et al; licensee BioMed Central Ltd.

This is an Open Access article distributed under the terms of the Creative Commons Attribution License (http://creativecommons.org/licenses/by/2.0), which permits unrestricted use, distribution, and reproduction in any medium, provided the original work is properly cited.

\begin{abstract}
Background: Legionella species cause severe forms of pneumonia with high mortality and complication rates. Accurate clinical predictors to assess the likelihood of Legionella communityacquired pneumonia (CAP) in patients presenting to the emergency department are lacking.
\end{abstract}

Methods: We retrospectively compared clinical and laboratory data of 82 consecutive patients with Legionella CAP with 368 consecutive patients with non-Legionella CAP included in two studies at the same institution.

Results: In multivariate logistic regression analysis we identified six parameters, namely high body temperature (OR I.67, p < $0.000 \mathrm{I}$ ), absence of sputum production (OR 3.67, $\mathrm{p}<0.000 \mathrm{I}$ ), low serum sodium concentrations (OR 0.89, $\mathrm{P}=0.0 \mathrm{II})$, high levels of lactate dehydrogenase (OR I.003, $\mathrm{p}=0.007)$ and $\mathrm{C}$-reactive protein $(\mathrm{OR} \mathrm{I} .006, \mathrm{p}<0.000 \mathrm{I})$ and low platelet counts $(\mathrm{OR} 0.99 \mathrm{I}, \mathrm{p}<$ $0.000 \mathrm{I}$ ), as independent predictors of Legionella CAP. Using optimal cut off values of these six parameters, we calculated a diagnostic score for Legionella CAP. The median score was significantly higher in Legionella CAP as compared to patients without Legionella (4 (IQR 3-4) vs 2 (IQR I-2), P $<0.000 \mathrm{I})$ with a respective odds ratio of $3.34(95 \% \mathrm{Cl} 2.57-4.33, \mathrm{p}<0.000 \mathrm{I})$. Receiver operating characteristics showed a high diagnostic accuracy of this diagnostic score (AUC $0.86(95 \% \mathrm{Cl} 0.8 \mathrm{I}-$ 0.90 ), which was better as compared to each parameter alone. Of the 191 patients (42\%) with a score of 0 or I point, only $3 \%$ had Legionella pneumonia. Conversely, of the 73 patients (16\%) with $\geq 4$ points, $66 \%$ of patients had Legionella CAP.

Conclusion: Six clinical and laboratory parameters embedded in a simple diagnostic score accurately identified patients with Legionella CAP. If validated in future studies, this score might aid in the management of suspected Legionella CAP. 


\section{Background}

Legionella species (spp.) causes a severe form of community-acquired pneumonia (CAP) with a high incidence of adverse medical outcomes including progression of infiltrates, respiratory failure and need for intensive care unit (ICU) admission [1,2]. In addition, Legionella CAP has a high mortality rate of about 10 percent, which may increase up to 27 percent in patients not receiving adequate antibiotics as part of the empiric treatment on admission [2]. Early identification of Legionella spp. in patients presenting with respiratory symptoms and suspicion of CAP to the emergency department is thus of utmost importance because it affects the timing and choice of empirical antibiotic therapy and reduces the risk for adverse outcome. Currently available diagnostic tests include detection of Legionella spp. by culture or polymerase chain-reaction (PCR) in respiratory samples and Legionella pneumophila antigen testing in urine. These tests lack sensitivity, in addition the urine antigen test only identifies Legionella pneumophila serogroup 1 [3,4]. Previous studies comparing clinical, radiological and laboratory findings in Legionella CAP and non-Legionella CAP have produced controversial results [1,5-12]. Two previous attempts to construct a diagnostic score that identifies Legionella in patients with CAP have been disappointing [1,5-11]. As a limitation, these studies compared Legionella pneumophila CAP with selected cases of pneumococcal CAP $[5,6,8]$. When applied in unselected patients, these scores lack sensitivity and/or specificity. Because of this diagnostic dilemma consensus guidelines on empiric antibiotic therapy for patients with CAP to extend antibiotic coverage to Legionella in all patients with severe CAP contributing to antibiotic overuse and emergence of multi-resistant strains [13-16]. However more recent findings suggest, that severity of CAP is not an appropriate screening criterion for Legionella CAP, which further complicates the choice of empiric antibiotic treatment [4].

The aim of this study was to compare initial clinical and laboratory parameters of consecutive patients with Legionella CAP who were hospitalized in our institution during the last 10 years with patients with non-Legionella CAP included in two studies at the same institution $[17,18]$ and thereby to identify reliable diagnostic predictors of Legionella CAP.

\section{Methods}

\section{Setting and Study population}

We retrospectively evaluated all consecutive patients who were admitted to the University Hospital in Basel, Switzerland from 1997 to 2007, with a diagnosis of Legionella CAP. The diagnosis of Legionella was considered definite if Legionella was either isolated by culture or PCR of a respiratory sample or detected by urinary antigen testing.
Patient records were reviewed with a standardized datacollection form to retrieve all demographic, clinical, microbiological, radiographic, laboratory and therapeutical data. To achieve a reasonable comparison, we used clinical and laboratory data on admission to the emergency department.

For comparison we used data on admission of a consecutive cohort of 368 patients with non-Legionella CAP who were admitted between December 2002 through February 2005 to the same institution and enrolled in two studies $[17,18]$. The design of the two studies was similar and has been reported in detail elsewhere $[17,18]$. In brief, a total of 373 consecutive patients with radiologically proven CAP were randomly assigned to be treated either with a procalcitonin (PCT)-based algorithm or standard practice. The primary endpoint of the two studies was to evaluate antibiotic exposure of a PCT-guided treatment algorithm as compared to standard recommended guidelines [14]. Both studies excluded patients with cystic fibrosis or active pulmonary tuberculosis, hospital-acquired pneumonia and severely immunocompromized patients. In both studies, testing for Legionella with the use of the urine antigen test was recommended in all CAP patients as part of the hospital work up for CAP and part of the study protocols. Investigation for other atypical bacterial pathogens namely Mycoplasma spp. and Chlamydia spp. were only tested in suspected cases upon the decision of to the treating physicians. For the purpose of this study, the five enrolled patients with Legionella CAP were analyzed in the Legionella CAP group.

The two intervention studies were previously approved by the local Ethical Committee (EKBB, Ethikkommission beider Basel) and registered in the current controlled trials databases. The Institutional Review Board classified this retrospective analysis as a quality control study and waived the need for informed consent.

CAP was defined as the presence of an infiltrate on chest radiograph and at least one of the following signs and symptoms (cough, sputum production, dyspnea, core body temperature $>38.0^{\circ} \mathrm{C}$, auscultatory findings of abnormal breath sounds and rales [19]. In all patients the Pneumonia Severity Index (PSI) and the CURB65 were calculated based on patients unique set of variables as described in detail elsewhere $[20,21]$. The goldstandard for diagnosis of Legionella CAP was the clinical diagnosis of a community-acquired pneumonia with an infiltrate on chest X-ray and at least one positive microbiological test for Legionella (urinary antigen testing, culture results from respiratory specimen or PCR from respiratory specimen). 


\section{Laboratory assessment}

For our analysis, we used laboratory results including markers of infection (white blood count (WBC) and Creactive protein (CRP)), liver enzymes (ALAT, ASAT, $\gamma$ glutamyltranspeptidase, alkaline phosphatase, bilirubin), markers of renal function (creatinine, urea), electrolytes (sodium, potassium), urinary testing for proteinuria and hemoglobinuria and results from arterial blood gas analysis $(\mathrm{pH}, \mathrm{PaO} 2)$ from the routinely collected blood analysis of all patients on admission. In our hospital, CRP concentrations are determined by an enzyme immunoassay having a detection limit of $<5 \mathrm{mg} / \mathrm{dl}$ (EMIT, Merck Diagnostica, Zurich, Switzerland).

In the group of Legionella CAP Legionella spp. was diagnosed either by antigen in urine detection (Legionella pneumophila serogroup 1) using an immunoenzymetric commercial method (Legionella Urinary Antigen; Binax), by PCR from respiratory samples using an in-house PCR technique from the Institute for Medical Microbiology (IMM) in Zurich, Switzerland, or by culture [22,23].

\section{Statistical analysis}

Discrete variables are expressed as counts (percentage) and continuous variables as medians and interquartile ranges (IQR). Frequency comparison was done by chisquare test. Two-group comparison of normally distributed data was performed by Students t-test. For data not normally distributed, the Mann-Whitney-U test was used. To estimate the potential clinical relevance of laboratory and clinical parameters to diagnose Legionella CAP, we used likelihood-ratio tests and univariate and multivariate logistic regression models. Thereby, outcomes were either Legionella CAP or non-Legionella CAP. For all independent variables in multivariate analysis, we calculated receiveroperating-characteristics (ROC) with the area under the ROC curve (AUC) being an overall diagnostic measure. For all calculations we used STATA 9.2 statistical software (Stata Corp, College Station, Tex). All testing was twotailed and P-values less than 0.05 were considered to indicate statistical significance.

\section{Results \\ Baseline parameters}

This pooled analysis includes 82 patients with the definite diagnosis of Legionella CAP and 368 patients with nonLegionella CAP. The median age of the pooled 450 patients was 72 years (IQR 58-81) and 62\% were males. Patients with Legionella CAP were significantly younger (68 (IQR 49-77) vs 73 (IQR 59-82), p =0.01), and had less frequent underlying chronic obstructive pulmonary disease (10\% vs $24 \%, \mathrm{p}=0.004)$ and heart disease $(24 \%$ vs $42 \%$, $\mathrm{p}=0.003)$. Cough $(70 \%$ vs $89 \%, \mathrm{p}<0.001)$, increase in sputum production (34\% vs $72 \%, \mathrm{p}<0.001)$ and dyspnea $(60 \%$ vs $72 \%$, p < 0.03), the cardinal symptoms of CAP, were more frequently found in non-Legionella CAP, while Legionella CAP patients had a higher median body temperature on presentation $\left(39.3^{\circ} \mathrm{C}\right.$ vs. $\left.38.4^{\circ} \mathrm{C}, \mathrm{p}<0.001\right)$. As demonstrated in Table 1, Legionella CAP patients had higher concentrations of CRP, lactate dehydrogenase $(\mathrm{LDH})$ and creatinine kinase $(\mathrm{CK})$, more frequently elevated liver enzymes, proteinuria and hemoglobinuria and lower concentrations of sodium, platelets and $\mathrm{pH}$ as compared to non-Legionella CAP patients. Additional demographic, clinical and laboratory data are presented in Table 1. Cough, increase of sputum production, dyspnea and confusion in patients with Legionella CAP were available in $96 \%(\mathrm{n}=79), 94 \%(\mathrm{n}=77), 94 \%(\mathrm{n}=77)$ and $96 \%(n=79)$, respectively.

The diagnosis of the 82 patients with Legionella CAP was established with a total of 164 microbiological tests including 72 urinary antigen tests, 54 cultures and 38 PCRs of respiratory specimen. Because in most patients multiple diagnostic tests were performed, addition did not sum up to $100 \%$. As demonstrated in Table 2, three different etiologic groups of Legionella were identified, namely $69.5 \%(\mathrm{n}=59)$ with only Legionella pneumophila serogroup $1,15.9 \%(\mathrm{n}=13)$ with Legionella pneumophila and Legionella species and $14.6 \%(\mathrm{n}=12)$ with only Legionella species, whereas in the previous two groups ( $\mathrm{n}$ $=13$ and $n=12$ ) serogroups and species of Legionella were not otherwise specified. Among patients who received urinary antigen testing, results for Legionella pneumophila serogroup 1 were positive in 59 of 72 patients $(82 \%)$. In patients with negative urinary antigen testing $(\mathrm{n}=13)$, diagnosis of Legionella was established by culture in 5 patients and/or PCR in 13 patients. In patients with no urine antigen testing $(\mathrm{n}=10)$ diagnosis was ascertained by culture and/or PCR. In the 368 patients with nonLegionella CAP urinary antigen testing for Legionella pneumophila serogroup 1 was performed with negative results. In these patients a causative microorganism in blood was found in $8.4 \%$ (Streptococcus pneumoniae (6.0\%), Staphylococcus aureus (0.8\%), Escherichia coli (0.4\%), Klebsiella pneumoniae $(0.2 \%)$ and others $(1.0 \%))$.

\section{Diagnostic reliability of clinical and laboratory parameters} To assess the diagnostic reliability of laboratory parameters and clinical signs to identify Legionella CAP patients, we calculated univariate logistic regression analysis (Table 3) with all parameters that were significantly different between Legionella and non-Legionella CAP patients on presentation. Further, to identify independent predictors of Legionella, we calculated multivariate logistic regression analysis (Table 3). Multivariate analysis identified two clinical parameters, namely body temperature (OR 1.67, p $<0.0001$ ), and absence of sputum production (OR 3.67, $\mathrm{p}<0.0001)$, and four laboratory parameters, namely sodium (OR 0.89, $\mathrm{p}=0.01$ ), LDH (OR 1.003, p = 0.007), 
Table I: Baseline Characteristics of the 450 Patients with CAP separated by Legionella CAP $(n=82)$ and non-Legionella CAP $(n=368)$.

\begin{tabular}{|c|c|c|c|}
\hline & $\begin{array}{l}\text { Legionella CAP } \\
\qquad(\mathrm{n}=82)\end{array}$ & $\begin{array}{c}\text { Non-Legionella CAP } \\
(\mathrm{n}=368)\end{array}$ & P-value \\
\hline \multicolumn{4}{|l|}{ Demographic characteristics } \\
\hline - Age (years)* & $68(49-77)$ & $73(59-82)$ & 0.01 \\
\hline - Sex (male) - no. (\%) & $57(70)$ & $221(60)$ & 0.11 \\
\hline \multicolumn{4}{|l|}{ Coexisting illnesses - no. (\%) } \\
\hline - Congestive heart disease & $20(24)$ & $156(42)$ & 0.003 \\
\hline - Cerebrovascular disease & $7(9)$ & $20(5)$ & 0.29 \\
\hline - Renal dysfunction & $23(28)$ & $100(27)$ & 0.15 \\
\hline- COPD $^{a}$ & $8(10)$ & $90(24)$ & 0.004 \\
\hline - Neoplastic disease & $17(21)$ & $53(14)$ & 0.15 \\
\hline \multicolumn{4}{|l|}{ Clinical History - no. (\%) } \\
\hline - Antibiotic pretreatment & $27(33)$ & $75(20)$ & 0.01 \\
\hline - Cough & $55(70)$ & $329(89)$ & $<0.001$ \\
\hline - increase in sputum production & $26(34)$ & $265(72)$ & $<0.001$ \\
\hline - Dyspnea & $46(60)$ & $266(72)$ & 0.03 \\
\hline \multicolumn{4}{|l|}{ Clinical and laboratory findings } \\
\hline - Confusion - no. (\%) & $9(11)$ & $31(8)$ & 0.4 \\
\hline - Respiratory rate (breaths/minute)* & $18(16-28)$ & $22(20-28)$ & 0.01 \\
\hline - Systolic blood pressure $(\mathrm{mmHg})^{*}$ & $130(\mid 13-145)$ & $130(1 \mid 3-143)$ & 0.9 \\
\hline - Heart rate (beats/minute)* & $100(86-114)$ & $95(80-110)$ & 0.05 \\
\hline - Body temperature $\left({ }^{\circ} \mathrm{C}\right)^{*}$ & $39.3(38.5-40.1)$ & $38.4(37.6-39.2)$ & $<0.001$ \\
\hline \multicolumn{4}{|l|}{ Laboratory findings } \\
\hline - C-reactive protein $(\mathrm{mg} / \mathrm{L})^{*}$ & $24 \mid(|7|-3 \mid 5)$ & $134(67-217)$ & $<0.001$ \\
\hline - Procalcitonin $(\mu \mathrm{g} / \mathrm{L})^{*}$ & $2.90(0.93-6.43)$ & $0.57(0.19-2.61)$ & $<0.01$ \\
\hline - Hematocrit (\%)* & $37(32-4 I)$ & $38(35-40)$ & 0.17 \\
\hline - Leucocyte counts $(\times 109 / \mathrm{L}) *$ & $12.9(9.5-15.5)$ & $12.8(8.9-16.8)$ & 0.47 \\
\hline - Platelet counts $(\times 109 / \mathrm{L})^{*}$ & $191(141-253)$ & $243(190-326)$ & $<0.001$ \\
\hline - Sodium $(\mathrm{mmol} / \mathrm{L})^{*}$ & $132(128-135)$ & $136(133-138)$ & $<0.001$ \\
\hline$<131 \mathrm{mmol} / \mathrm{L}-$ no. $(\%)$ & $36(46)$ & $49(14)$ & $<0.001$ \\
\hline - Creatinine (umol/L)* & $101(80-144)$ & $105(83-138)$ & 0.29 \\
\hline - Urea $(\mathrm{mmol} / \mathrm{L})^{*}$ & $8.3(5.4-14.2)$ & $6.9(4.9-11.5)$ & 0.93 \\
\hline - Elevated liver enzymes ${ }^{b}-$ no. (\%) & $52(63)$ & $126(34)$ & $<0.001$ \\
\hline - Creatinine kinase $(\mathrm{U} / \mathrm{L})^{*}$ & $103(48-417)$ & $77(4 I-174)$ & $<0.01$ \\
\hline - Lactate dehydrogenase $(\mathrm{U} / \mathrm{L})^{*}$ & $252(204-382)$ & $214(175-268)$ & $<0.001$ \\
\hline - Glucose (mmol/L)* & $6.9(6.1-9.7)$ & $6.8(5.9-8.4)$ & 0.12 \\
\hline - Oxygen saturation (\%)* & 93 (88-95) & $93(89-96)$ & 0.46 \\
\hline - $\mathrm{PaO} 2(\mathrm{kPa})^{*}$ & $7.7(7.1-9.9)$ & $8.0(6.8-9.5)$ & 0.82 \\
\hline$-\mathrm{pH}^{*}$ & $7.46(7.38-7.49)$ & $7.43(7.39-7.46)$ & 0.02 \\
\hline - Hemoglobinuria - no. (\%) & $45(70)$ & $114(45)$ & $<0.001$ \\
\hline - Proteinuria - no. (\%) & $46(73)$ & $124(48)$ & $<0.001$ \\
\hline \multicolumn{4}{|l|}{ Risk assessment } \\
\hline - PSIc Points* & $117(90-140)$ & $95(73-121)$ & $<0.001$ \\
\hline - CURB65* & $2(I-2)$ & I (I-2) & 0.09 \\
\hline \multicolumn{4}{|l|}{ Outcome - no. (\%) } \\
\hline - Death & $13(16)$ & $33(9)$ & 0.06 \\
\hline - Admission to ICUd & $34(4 I)$ & $43(12)$ & 0.01 \\
\hline - Length of hospital stay (days)* & $13(8-20)$ & $11(6-17)$ & 0.02 \\
\hline
\end{tabular}

* denotes median (interquartile range); a COPD denotes chronic obstructive pulmonary disease; b elevated liver enzymes denotes elevation of ASAT and/or ALAT; c PSI denotes Pneumonia Severity Index; d ICU denotes intensive care unit.

CRP (OR 1.006, p < 0.0001) and platelet counts (OR $0.991, \mathrm{p}<0.0001$ ) as independent predictors of Legionella $\mathrm{CAP}$, respectively. To estimate the clinical usefulness of these parameters and to compare sensitivities and specificities, ROC curves using these 6 predictors were calculated
(Figure 1). The area under the ROC curve, optimal cut off values and corresponding sensitivities and specificities are presented in table 4 for each variable at the optimal cut off point are presented in Table 4. Using these optimal cut off values, we calculated a 6 point diagnostic score giving a 
Table 2: Results of microbiological tests $(n=164)$ in all patients with Legionella CAP $(n=82)$.

\begin{tabular}{|c|c|c|c|c|}
\hline Established by IEtiologic Diagnosis & & $\begin{array}{l}\text { L. pn Ia } \\
(\mathrm{n}=57)\end{array}$ & $\begin{array}{c}\text { L. pnb \& L. } \\
\text { spp.c } \\
(n=\mid 3)\end{array}$ & $\begin{array}{l}\text { L. spp } \\
(\mathrm{n}=12)\end{array}$ \\
\hline \multirow[t]{2}{*}{ Urinary antigen testing $(n=72)$} & Positive $(n=59)$ & 50 & 9 & 0 \\
\hline & Negative $(n=13)$ & 1 & 4 & 8 \\
\hline \multirow{2}{*}{$\begin{array}{l}\text { Respiratory specimen } \\
\text { culture }(n=54)\end{array}$} & Positive $(n=23)$ & 14 & 8 & 1 \\
\hline & Negative $(n=3 I)$ & 19 & 5 & 7 \\
\hline \multirow{2}{*}{$\begin{array}{l}\text { Respiratory specimen } \\
\operatorname{PCR}^{d}(n=38)\end{array}$} & Positive $(n=34)$ & 10 & 13 & 11 \\
\hline & Negative $(n=4)$ & 4 & 0 & 0 \\
\hline
\end{tabular}

a L. pn I, b L. pn and c L. spp. denote Legionella pneumophila serogroup I, Legionella pneumophila and Legionella species; d PCR denotes polymerase chain-reaction, respectively.

The goldstandard for diagnosis of Legionella CAP was the clinical diagnosis of a community-acquired pneumonia with an infiltrate on chest X-ray and at least one positive microbiological test for Legionella (urinary antigen testing, culture results from respiratory specimen or PCR from respiratory specimen).

point for each variable beyond the optimal cut off. Patients with Legionella CAP had a significantly higher median score as compared to patients without Legionella (4 (IQR 3-4) vs 2 (IQR $1-2$ ), p < 0.0001). The odds ratio of this score to predict Legionella was 3.34 (95\%CI 2.57$4.33, \mathrm{p}<0.0001$ ). With an area under the curve (AUC) of

Table 3: Prediction of Legionella CAP $(n=82)$ in univariate (a) and multivariate (b) logistic regression analysis

\begin{tabular}{|c|c|c|c|c|}
\hline Univariate analysis & & & & \\
\hline Predictor & Odds ratio & \multicolumn{2}{|c|}{$\left(95 \% \mathrm{Cl}^{*}\right)$} & $\mathbf{P}$-value \\
\hline Age & 0.98 & 0.97 & 1.00 & 0.017 \\
\hline Temperature & 1.93 & $|.5|$ & 2.46 & $<0.0001$ \\
\hline No dyspnea & 1.75 & 1.06 & 2.9 & 0.03 \\
\hline No cough & 3.7 & 2.05 & 6.6 & $<0.0001$ \\
\hline No sputum & 5.14 & 3.04 & 8.7 & $<0.0001$ \\
\hline Sodium & 0.86 & 0.82 & 0.91 & $<0.0001$ \\
\hline Elevated liver enzymes' & 3.32 & 2.02 & 5.48 & $<0.0001$ \\
\hline Lactate dehydrogenase & 1.003 & 1.002 & 1.005 & 0.001 \\
\hline Creatinine kinase & 1.001 & 1.000 & 1.001 & 0.001 \\
\hline C-reactive protein (CRP) & 1.006 & 1.004 & 1.008 & $<0.0001$ \\
\hline Platelet counts & 0.993 & 0.990 & 0.996 & $<0.0001$ \\
\hline Hemoglobinuria & 2.95 & 1.64 & 5.32 & $<0.0001$ \\
\hline Proteinuria & 2.88 & 1.56 & 5.29 & 0.001 \\
\hline \multicolumn{5}{|l|}{ Multivariate analysis } \\
\hline Predictor & Odds ratio & \multicolumn{2}{|c|}{$(95 \% \mathrm{Cl})$} & P-value \\
\hline Temperature & 1.67 & 1.23 & 2.32 & $<0.0001$ \\
\hline No sputum & 3.67 & 1.8 & 7.4 & $<0.0001$ \\
\hline Sodium & 0.89 & 0.84 & 0.96 & 0.01 \\
\hline Lactate dehydrogenase & 1.003 & 1.000 & 1.005 & 0.007 \\
\hline C-reactive protein (CRP) & 1.006 & 1.003 & 1.009 & $<0.0001$ \\
\hline Platelet counts & 0.991 & 0.987 & 0.995 & $<0.0001$ \\
\hline
\end{tabular}

* Cl denotes confidence interval; I elevated liver enzymes denotes ASAT and/or ALAT.
$0.86(95 \%$ CI $0.81-0.90)$, the diagnostic accuracy of this diagnostic score was significantly better as compared to each single one of the six parameters. Table 5 shows corresponding sensitivities, specificities and the number of patients with and without Legionella CAP for each cut off point of the diagnostic score. In Figure 2 the distribution of Legionella and non-Legionella CAP according to the score is demonstrated. Of the 191 patients (42\%) with 0 or 1 point, only $6(3 \%)$ had Legionella pneumonia. Conversely, of the 73 patients $(16 \%)$ with $\geq 4$ points, 48 patients (66\%) had Legionella CAP.

\section{Discussion}

The timely identification of Legionella in patients presenting with CAP to the emergency department is challenging because single clinical and laboratory parameters have shown low sensitivity and/or specificity. A reliable diagnostic score integrating different parameters is lacking [8]. The results of this study indicate that six clinical and laboratory parameters, namely high body temperature, absence of sputum production, low serum sodium and platelet counts, and high LDH and CRP concentrations combined in a diagnostic score reliably estimate the likelihood of Legionella in patients who present with CAP to the emergency department.

Current guidelines on the management of patients with CAP recommend that empirical antibiotic coverage should be extended to Legionella in "suspicious cases", although no single parameter can reliably identify or rule out patients at risk for Legionella $[8,13]$. Previous studies have addressed this dilemma and assessed potential predictors of Legionella CAP in different settings [1,5-12]. A Spanish study found that Legionella should be suspected in middle-aged, alcohol drinking, healthy male patients, 
Table 4: Area under the curve (AUC) of receiver operating curve (ROC) characteristic plot analysis.

\begin{tabular}{|c|c|c|c|c|c|c|}
\hline Parameter & AUC & $95 \% \mathrm{Cl}^{*}$ & P-value & Optimal cut off & Sensitivity & Specificity \\
\hline Combined Score & 0.86 & $0.8 I-0.90$ & $\cdot$ & $<2$ & 78.0 & 78.8 \\
\hline Temperature & 0.74 & $0.63-0.78$ & $<0.0001$ & $>39.4$ & 48.1 & 84.4 \\
\hline No sputum & 0.68 & $0.6 I-0.74$ & $<0.0001$ & - & - & - \\
\hline Sodium & 0.71 & $0.63-0.78$ & $<0.0001$ & $<133$ & 64.6 & 70.8 \\
\hline Lactate dehydrogenase & 0.62 & $0.53-0.71$ & $<0.0001$ & $>225$ & 67.1 & 58.1 \\
\hline C-reactive protein & 0.76 & $0.70-0.82$ & $<0.0001$ & $>187$ & 71.6 & 64.7 \\
\hline Platelet counts & 0.71 & $0.64-0.78$ & $<0.0001$ & $<17 \mid$ & 45.7 & 83.6 \\
\hline
\end{tabular}

$* \mathrm{Cl}$ denotes confidence interval;

if patients lack response to previous beta-lactamic drugs, if headache, diarrhea, severe hyponatremia, and elevation in serum CK levels were present, or if no cough, expectoration, and thoracic pain were found [12]. A recent critical review included 13 different studies that reported clinical details for the evaluation of Legionella CAP, however, concluded that using a syndromic approach cannot be recommended and, thus, an empiric antibiotic therapy covering for Legionella for all patients with CAP requiring hospitalization should be recommended [8]. This, however, results in indiscriminate use of, mostly unnecessary, dual antibiotic therapy and thus, increased antibiotic exposure and emergence of resistance. Two previous studies have proposed a clinical prediction rule to identify Legionella CAP $[5,6]$. The CBPIS score has been proposed with a maximum of 17 points based on the evaluation of temperature, serum creatinine, sodium and LDH concentrations, headache, vomiting and smoking. However, in a prospective validation study, this scoring system had a low sensitivity and/or specificity to diagnose or exclude Legionella CAP [5]. Guidance of antibiotic therapy using this score would have left half of the patients with Legionella CAP without specific coverage and would have led to the administration of an unnecessarily broad antibiotic regimen in $14 \%$ of patients without Legionella. In addition, the model was unable to distinguish between Legionella pneumophila and Streptococcus pneumoniae, because the majority of patients were categorized into the "intermediate-probability" group. Similarly, the clinical criteria proposed by the Winthrop-University Hospital (WUH) for the identification of Legionellosis showed an inadequately low sensitivity and specificity of $78 \%$ [6]. In addition, the WUH study included clinical data collected throughout the first 7 days of hospitalization limiting its use as a screening tool in an emergency department setting. Importantly, both studies based their scoring system on the comparison of Legionella CAP with pneumococcal $\mathrm{CAP}$, which does not reflect the clinical situation in the emergency department, where the decision about empirical antibiotic therapy must be taken in all patients with CAP. In this study, a thorough and comprehensive 10 year retrospective data collection was conducted and data from two prospective studies presenting to the emergency department of the same institution were used to compare clinical and laboratory parameters. Thus we believe the proposed score reflects the clinical situation more realistically.

Simplicity of a diagnostic score is a major determinant for its future usefulness in daily practice. The WUH scale consists of 23 different criteria, which increases its complexity and may correlate with non-adherence as observed in clinical severity prediction scores $[6,24]$. The herein proposed scoring system composes of only 6 routinely measured clinical and laboratory parameters that showed an independent predictive value in multivariate logistic regression analysis and a high overall diagnostic performance as demonstrated in ROC analysis.

As with any diagnostic score, the "optimal" cut off should be chosen based on the pretest probability and the clinical context, particularly the risk for adverse medical outcomes

Table 5: Diagnostic accuracy of the diagnostic score at different cut off points.

\begin{tabular}{ccccc}
\hline Points & Sensitivity & Specificity & Legionella CAP & Non-Legionella CAP \\
\hline$\geq 0$ & 100 & - & $0(0 \%)$ & $70(19.0 \%)$ \\
$\geq 1$ & 92.7 & 50.3 & $6(7.3 \%)$ & $115(31.3 \%)$ \\
$\geq 2$ & 78.0 & 78.8 & $12(14.6 \%)$ & $105(28.5 \%)$ \\
$\geq 3$ & 58.5 & 93.2 & $16(19.5 \%)$ & $53(14.4 \%)$ \\
$\geq 4$ & 14.6 & 98.9 & $36(43.9 \%)$ & $21(5.7 \%)$ \\
$\geq 5$ & 2.4 & 100 & $10(12.2 \%)$ & $4(1.1 \%)$ \\
6 & - & 100 & $2(2.4 \%)$ & $0(0 \%)$ \\
\hline
\end{tabular}




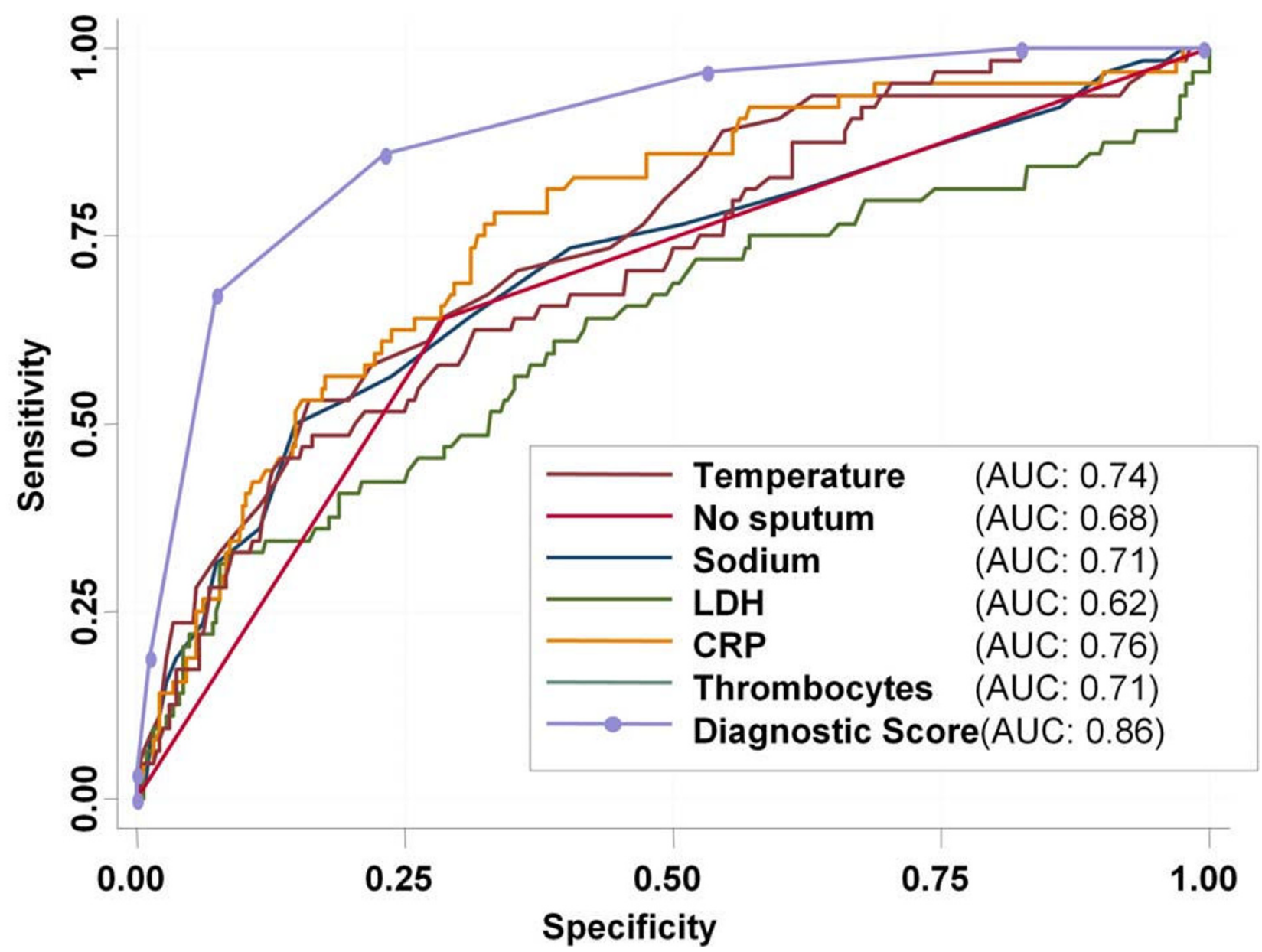

Figure I

Receiver Operating Curve (ROC) analysis of different clinical and laboratory parameters to differentiate Legionella CAP from non-Legionella CAP. AUC denotes area under the curve; LDH and CRP denote lactate dehydrogenase and C-reactive protein.

of patients. Supported by recent guidelines, treatment decision on the empirical antibiotic therapy should be based on both, the diagnostic probability and the risk of patients based on a prognostic assessment. Importantly, the PSI - but not the CURB65 score - showed a high prognostic accuracy to predict adverse medical outcomes in patients with Legionella CAP in this study. This finding is reassuring because prognostic CAP scores mainly depend on age and Legionella CAP patients tend to be younger. Thus, taking the diagnostic limitations of urinary antigen testing into account, empirical antibiotic therapy covering for atypical pathogens in patients with negative urinary antigen testing but high diagnostic probability (diagnostic score $\geq 4$ ) may be advisable. Conversely, in low risk patients with low diagnostic probability (diagnostic score $\leq 1$ ) and no clinical or diagnostic evidence of CAP caused by other atypical pathogens, macrolides may initially be withheld until the results of the urinary antigen tests become available.

Some limitations should be considered in interpreting our results. With a retrospective design, our results are preliminary and prospective validation is needed prior to a widespread use in clinical practice. The diagnosis of Legionella spp. in non-Legionella CAP patients was performed routinely by urinary antigen testing without PCR, culture or serology and we may have classified some patients incorrectly. Moreover there was no further routinely investigation for other atypical bacterial pathogens. We chose the 10 year retrospective design to provide the necessary number of Legionella cases to calculate multivariate analysis with adequate power. The proposed score consists of 


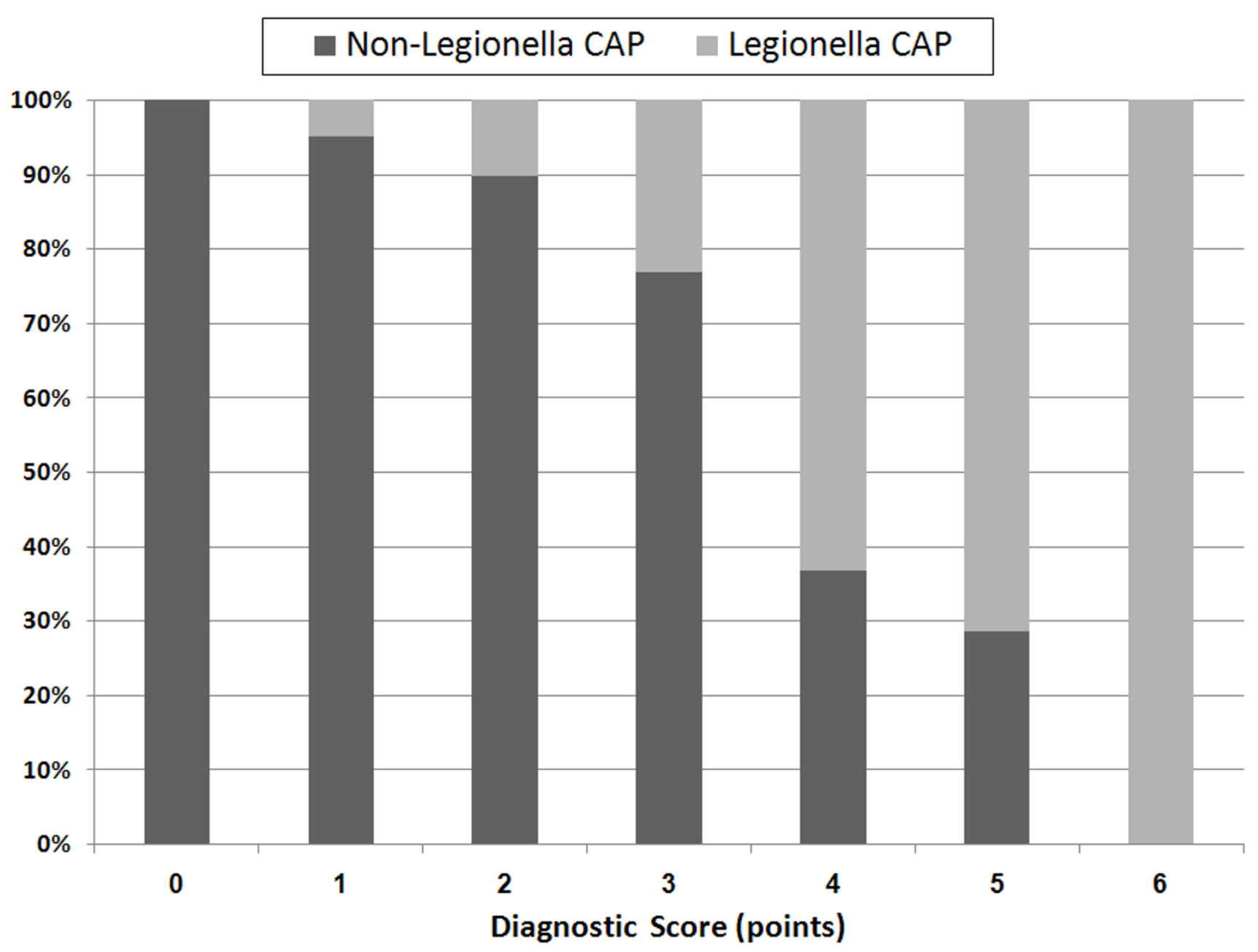

Figure 2

Patients with non-Legionella CAP (dark gray) and Legionella CAP (light gray) according to the points of the diagnostic score. Numbers in the boxes represent total number of patients.

mainly objectively measurable parameters, which may robustly be ascertained in retrospect. Still, anamnestic parameters (i.e. absence of sputum production), even if highly available, may not be accurately assessed in retrospect and are thus underestimated in favour of laboratory parameters and clinical data.

\section{Conclusion}

In conclusion, the results of this study suggest that six clinical and laboratory signs embedded in a simple diagnostic score allow better differentiation of Legionella from unselected patients with non-Legionella CAP presenting to the emergency department. If confirmed in prospective studies, this score might improve the timing and choice of empirical antibiotic therapy and thus reduce the associated morbidity and mortality and the emergence of bacterial resistance.

\section{Competing interests}

The authors declare that they have no competing interests.

\section{Authors' contributions}

PS, BM, RF, RZ, and JH had the idea for the study and directed the study design. PS, BM, MCC, RF, RZ, JH, AT, RF and IS directed the data collection. PS RF, RZ and JH analyzed the data and wrote the first report. PS, BM, MCC, RF, $\mathrm{RZ}, \mathrm{JH}, \mathrm{AT}, \mathrm{RF}$ and IS had substantial contributions in planning of the study, data collection, interpretation of data and/or writing of the manuscript.

\section{Acknowledgements}

We thank the staff of the clinics of emergency medicine, internal medicine and endocrinology and the department of clinical chemistry, notably Fausta Chiaverio, Martina-Barbara Bingisser, Maya Kunz, Vreni Wyss and Ursula Schild, for most helpful support during the study. 


\section{References}

I. Stout JE, Yu VL: Legionellosis. N Engl J Med I997, 337:682-687.

2. Falco V, Fernandez de Sevilla T, Alegre J, Ferrer A, Martinez Vazquez JM: Legionella pneumophila. A cause of severe communityacquired pneumonia. Chest 1991, 100:1007-1011.

3. Kohler RB, Winn WC Jr, Wheat LJ: Onset and duration of urinary antigen excretion in Legionnaires disease. J Clin Microbio 1984, 20:605-607.

4. von Baum H, Ewig S, Marre R, Suttorp R, Gonschior S, Welte T, Luck $C:$ Community-Acquired Legionella Pneumonia: New Insights from the German Competence Network for Community Acquired Pneumonia. Clin Infect Dis 2008, 46: I356-64.

5. Fernandez-Sabe N, Roson B, Carratala J, Dorca J, Manresa F, Gudiol F: Clinical diagnosis of Legionella pneumonia revisited: evaluation of the Community-Based Pneumonia Incidence Study Group scoring system. Clin Infect Dis 2003, 37:483-489.

6. Gupta SK, Imperiale TF, Sarosi GA: Evaluation of the WinthropUniversity Hospital criteria to identify Legionella pneumonia. Chest 2001, I20:1064-107I.

7. Fernandez JA, Lopez P, Orozco D, Merino J: Clinical study of an outbreak of Legionnaire's disease in Alcoy, Southeastern Spain. Eur J Clin Microbiol Infect Dis 2002, 2 I:729-735.

8. Mulazimoglu L, Yu VL: Can Legionnaires disease be diagnosed by clinical criteria? A critical review. Chest 200I, I 20:1049-1053.

9. Cunha BA: The atypical pneumonias: clinical diagnosis and importance. Clin Microbiol Infect 2006, I 2(Suppl 3): I 2-24

10. Cunha BA: Atypical pneumonias. Clinical diagnosis and empirical treatment. Postgrad Med I991, 90:89-90. 95-88, I0I

II. Sopena N, Pedro-Botet L, Mateu L, Tolschinsky G, Rey-Joly C, Sabria $M$ : Community-acquired legionella pneumonia in elderly patients: characteristics and outcome. J Am Geriatr Soc 2007 55:114-119.

12. Sopena N, Sabria-Leal M, Pedro-Botet ML, Padilla E, Dominguez J, Morera J, Tudela P: Comparative study of the clinical presentation of Legionella pneumonia and other communityacquired pneumonias. Chest 1998, I I3:I I95-I200.

13. Mandell LA, Wunderink RG, Anzueto A, Bartlett JG, Campbell GD Dean NC, Dowell SF, File TM Jr, Musher DM, Niederman MS, Torres A, Whitney CG, Infectious Diseases Society of America; American Thoracic Society: Infectious Diseases Society of America/ American Thoracic Society consensus guidelines on the management of community-acquired pneumonia in adults. Clin Infect Dis 2007, 44(Suppl 2):S27-72.

14. Woodhead M, Blasi F, Ewig S, Huchon G, Leven M, Ortqvist A, Schaberg T, Torres A, Heijden G van der, Verheij T]: Guidelines for the management of adult lower respiratory tract infections. Eur Respir J 2005, 26: I I38- I I80.

15. Fang GD, Fine M, Orloff J, Arisumi D, Yu VL, Kapoor W, Grayston JT, Wang SP, Kohler R, Muder RR, et al.: New and emerging etiologies for community-acquired pneumonia with implications for therapy. A prospective multicenter study of 359 cases. Medicine (Baltimore) 1990, 69:307-316.

16. Guillemot D, Courvalin P: Better control of antibiotic resistance. Clin Infect Dis 200I, 33:542-547.

17. Christ-Crain M, Stolz D, Bingisser R, Muller C, Miedinger D, Huber PR, Zimmerli W, Harbarth S, Tamm M, Muller B: Procalcitonin Guidance of Antibiotic Therapy in Community-acquired Pneumonia: A Randomized Trial. Am J Respir Crit Care Med 2006, 1 74:84-93.

18. Christ-Crain M, Jaccard-Stolz D, Bingisser R, Gencay MM, Huber PR, Tamm M, Muller B: Effect of procalcitonin-guided treatment on antibiotic use and outcome in lower respiratory tract infections: cluster-randomised, single-blinded intervention trial. Lancet 2004, 363:600-607.

19. Niederman MS, Mandell LA, Anzueto A, Bass JB, Broughton WA, Campbell GD, Dean N, File T, Fine MJ, Gross PA, Martinez F, Marrie T], Plouffe JF, Ramirez J, Sarosi GA, Torres A, Wilson R, Yu VL, American Thoracic Society: Guidelines for the management of adults with community-acquired pneumonia. Diagnosis, assessment of severity, antimicrobial therapy, and prevention. Am J Respir Crit Care Med 200I, I 63:1730-1754

20. Fine MJ, Auble TE, Yealy DM, Hanusa BH, Weissfeld LA, Singer DE, Coley CM, Marrie TJ, Kapoor WN: A prediction rule to identify low-risk patients with community-acquired pneumonia. $N$ Engl J Med 1997, 336:243-250.
21. Lim WS, Eerden MM van der, Laing $R$, Boersma WG, Karalus N Town GI, Lewis SA, Macfarlane JT: Defining community acquired pneumonia severity on presentation to hospital: an international derivation and validation study. Thorax 2003, 58:377-382.

22. Dominguez JA, Gali N, Pedroso P, Fargas A, Padilla E, Manterola JM, Matas L: Comparison of the Binax Legionella urinary antigen enzyme immunoassay (EIA) with the Biotest Legionella Urin antigen EIA for detection of Legionella antigen in both concentrated and nonconcentrated urine samples. J Clin Microbiol 1998, 36:2718-2722.

23. Lee TC, Vickers RM, Yu VL, Wagener MM: Growth of 28 Legionella species on selective culture media: a comparative study. J Clin Microbiol 1993, 3 I :2764-2768.

24. Lee RW, Lindstrom ST: A teaching hospital's experience applying the Pneumonia Severity Index and antibiotic guidelines in the management of community-acquired pneumonia. Respirology 2007, I 2:754-758.

\section{Pre-publication history}

The pre-publication history for this paper can be accessed here:

http://www.biomedcentral.com/1471-2466/9/4/prepub
Publish with BioMed Central and every scientist can read your work free of charge

"BioMed Central will be the most significant development for disseminating the results of biomedical research in our lifetime. "

Sir Paul Nurse, Cancer Research UK

Your research papers will be:

- available free of charge to the entire biomedical community

- peer reviewed and published immediately upon acceptance

- cited in PubMed and archived on PubMed Central

- yours - you keep the copyright
BioMedcentral 